\title{
Dynamic Surface Site Activation: A Rate Limiting Process in Electron Beam Induced Etching
}

\author{
Aiden A. Martin, Matthew R. Phillips, and Milos Toth* \\ School of Physics and Advanced Materials, University of Technology, Sydney, P.O. Box 123, \\ Broadway, New South Wales 2007, Australia \\ E-mail: milos.toth@uts.edu.au
}

\begin{abstract}
We report a new mechanism that limits the rate of electron beam induced etching (EBIE). Typically, the etch rate is assumed to scale directly with the precursor adsorbate dissociation rate. Here we show that this is a special case, and that the rate can instead be limited by the concentration of active sites at the surface. Novel etch kinetics are expected if surface sites are activated during EBIE, and observed experimentally using the electron sensitive material ultra nano-crystalline diamond (UNCD). In practice, etch kinetics are of interest because they affect resolution, throughput, proximity effects and the topography of nanostructures and nanostructured devices fabricated by EBIE.
\end{abstract}

Keywords: electron beam induced etching, ultra nano-crystalline diamond, reaction kinetics, defect generation, nanofabrication, surface chemistry

\section{Introduction}

Gas-mediated electron beam induced etching (EBIE) $)^{1-3}$ is a direct write nanolithography technique used to modify surfaces at nano- and micro-scales. EBIE proceeds through chemical re-

${ }^{*}$ To whom correspondence should be addressed 
actions induced by electron irradiation of a solid substrate exposed to a precursor gas. Surfaceadsorbed precursor molecules such as $\mathrm{H}_{2} \mathrm{O}$ are dissociated by electrons, generating fragments (e.g., $\mathrm{O}^{*}$ and $\left.\mathrm{OH}^{*}\right)^{4}$ that react with a substrate (e.g., C) to produce volatile species (e.g., $\mathrm{CO}$ and $\mathrm{CO}_{2}$ ) that desorb and are removed by a pumping system, thus giving rise to localized chemical dry etching in the vicinity of an electron beam (see Figure 1). Precursors such as $\mathrm{XeF}_{2}, \mathrm{Cl}_{2}$, $\mathrm{ClF}_{3}, \mathrm{NH}_{3}, \mathrm{O}_{2}$ and $\mathrm{H}_{2} \mathrm{O}$ can be used to etch a wide range of materials including graphene, carbon nanotubes, amorphous carbon, ${ }^{5-12}$ diamond ${ }^{13-15}$ and a variety metals, semiconductors and insulators. ${ }^{1-3}$ Nanometer resolution is attainable ${ }^{3}$ and $\sim 4 \mathrm{~nm}$ has been demonstrated in $\mathrm{H}_{2} \mathrm{O}$-mediated EBIE of carbon nanowires on electrically insulating, bulk quartz substrates. ${ }^{5}$ The technique is analogous to gas-assisted focused ion beam (FIB) milling. ${ }^{3,16}$ However, EBIE is a chemical process that does not involve sputtering or ion implantation.

EBIE resolution and the time-evolution of structures fabricated by EBIE are affected by the electron flux profile at the substrate surface, and by the precursor adsorbate supply and dissociation rates. $^{2,11,17}$ The flux profile is defined by the diameter and shape of the electron beam, and the spatial distribution of electrons emitted from the substrate. It governs EBIE resolution in the limit of zero depletion (i.e., in the so-called 'reaction rate limited' etch regime) where the etch rate scales linearly with electron flux. However, adsorbate depletion makes the etch rate sublinear with electron flux, which in turn serves to alter (usually decrease) resolution because the etch efficiency decreases with increasing electron flux which typically decreases with distance away from the electron beam axis. Consequently, much effort has gone into the development of simulators for predictive modeling of EBIE and the related technique of gas-mediated electron beam induced deposition (EBID). ${ }^{1-3,17-22}$ The models come in a number of varieties, but all are based on assumptions contained in rate equations of the form:

$$
\frac{\partial N_{a}}{\partial t}=\Lambda-k_{0} N_{a}-\frac{\partial N_{\alpha}}{\partial t}+D_{a} \nabla^{2} N_{a}
$$

where $a$ and $\alpha$ signify surface-adsorbed precursor molecules (e.g., $\mathrm{H}_{2} \mathrm{O}$ ) and fragments (e.g., $\mathrm{O}^{*}$ ), 


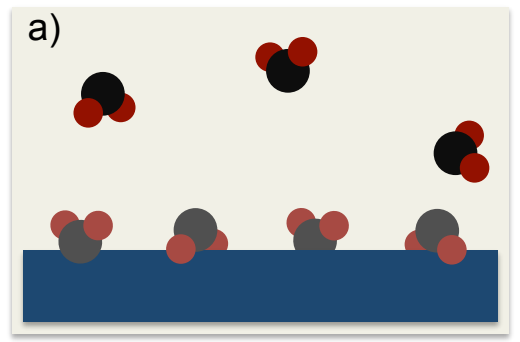

c)

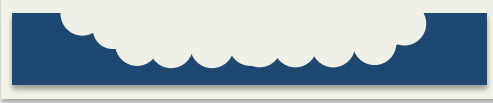

e)

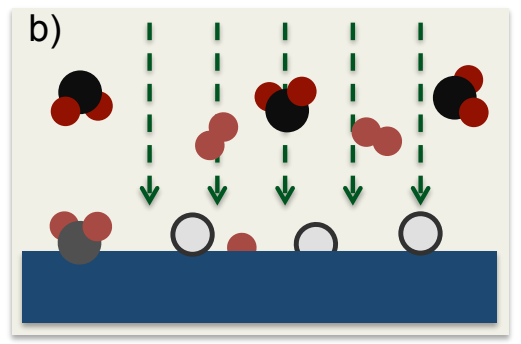

d)

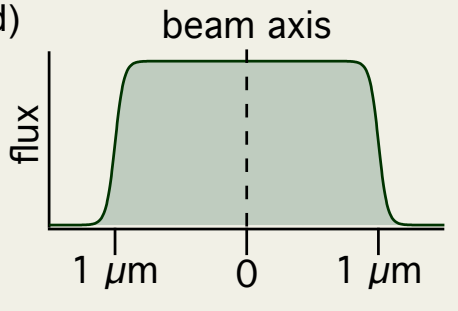

Figure 1: Simplified schematic of $\mathrm{H}_{2} \mathrm{O}$-mediated electron beam induced etching of carbon: (a) $\mathrm{H}_{2} \mathrm{O}$ adsorption and surface diffusion, (b) generation of $\mathrm{O}^{*}$ fragments by incident and emitted electrons, and (c) etching caused by $\mathrm{C}$ volatilization by $\mathrm{O}^{*}$ adsorbates. Also shown is (d) a $2 \mu \mathrm{m}$ wide electron beam with a top-hat flux profile, and (e) an AFM image of a pit etched in UNCD using a stationary top-hat beam (diameter $=2 \mu \mathrm{m}$, depth $\left(z_{d}\right)=175 \pm 22 \mathrm{~nm}$ ).

respectively, $\frac{\partial N_{a}}{\partial t}$ is the rate of change of concentration of precursor adsorbates at each point on the surface, expressed as a sum of fluxes $\left(\mathrm{m}^{-2} \mathrm{~s}^{-1}\right)$ representing adsorption $(\Lambda=s F(1-\Theta))$, desorption $\left(k_{0} N_{a}\right)$, electron induced dissociation $\left(\partial N_{\alpha} / \partial t\right)$ and diffusion $\left(D_{a} \nabla^{2} N_{a}\right) \cdot N$ is number density at the surface, $F$ is the gas molecule flux incident onto the substrate, $s$ is the sticking coefficient, $\Theta$ is $\mathrm{H}_{2} \mathrm{O}$ surface coverage ( $\Theta=A_{a} N_{a}$, and is typically limited to $1 \mathrm{ML}$ by the Langmuir isotherm), $A_{a}$ is the area of a single surface site, $k_{0}$ is the desorption rate and $D_{a}$ is the diffusion coefficient. The etch rate is given by:

$$
\begin{aligned}
& \frac{\partial N_{\alpha}}{\partial t}=\sigma_{\alpha} f N_{a}, \\
& \frac{\partial z_{d}}{\partial t}=V_{\gamma} \frac{\partial N_{\alpha}}{\partial t},
\end{aligned}
$$


where $f$ is electron flux, $\sigma_{\alpha}$ is the effective cross-section for the generation of fragments that volatilize the substrate, ${ }^{23} z_{d}$ is the depth of an etch pit such as the one in Figure 1(e), $\partial z_{d} / \partial t$ is the vertical etch rate and $V_{\gamma}$ is the volume of a single molecule (e.g., C) removed from the substrate in the etch reaction.

Eqs. (1) to (3), referred to from here on as 'model \#1', are representative of standard EBIE models, ${ }^{1-3,18,19}$ which are based on the assumption that the etch rate is proportional to the adsorbate dissociation rate (i.e., Eqs. (2) and (3)). A shortcoming of these models is that they neglect the possibility that volatilization (i.e., etching) may occur only at sites that are chemically 'active', such as defects, and that the active site concentration may change during EBIE. This simplification is clearly inappropriate for beam sensitive materials which are altered by the electron beam used for EBIE.

Electron beam damage (or 'restructuring') is a common phenomenon encountered in materials such as carbon whose defect structure and bond hybridisation can be altered by electron irradiation. ${ }^{24-31}$ It is well known (from plasma and thermal etching studies) that the bond hybridisation ${ }^{32}$ and defect structure ${ }^{33-35}$ of carbon affect the surface volatilization efficiency. It is therefore reasonable to expect the EBIE efficiency of such materials to change with time as an electron beam creates surface defects during etching. To verify this hypothesis, we generalize model \#1 to account for active sites at the surface, and dynamic surface site activation occurring during EBIE ('model \#2'). Subsequently, model \#2 is adapted to the specific case of site activation caused by electron beam damage of the substrate ('model \#3') which is shown to be in excellent agreement with EBIE experiments performed using the electron sensitive material ultra nano-crystalline diamond (UNCD). 


\section{Methods and materials}

\section{Modeling}

Models \#1 - \#3 were implemented using numerical methods described elsewhere. ${ }^{3,17}$ The parameters $f$ and $F$ were measured directly, $s$ was fixed at unity, $k_{0} \approx 10^{13} \mathrm{~s}^{-1},{ }^{36} E_{a}=0.48 \mathrm{eV}$, $V_{\gamma} \approx 5.70 \AA^{3}$ (calculated using a density of $3.5 \mathrm{~g} / \mathrm{cm}^{2}$ ), and the area of a $\mathrm{H}_{2} \mathrm{O}$ molecule 37 $\sim 14.8 \AA^{2}$. All experiments were performed under conditions where adsorbate depletion is negligible. The parameter $D$ was therefore set to zero. ${ }^{3,22}$ Calculated $N_{a}(t)$ profiles confirmed that the extent of depletion was negligible $(<1 \%)$ under all conditions used in the present study (i.e., $\left.N_{a}[t \rightarrow \infty] / N_{a}[t=0] \approx 1\right)$. Hydrophilic and hydrophobic surface were treated identically in the etch model since adsorbate depletion was negligible in both cases (i.e., surface hydrophobicity affects only the etching of the first monolayer of UNCD).

\section{Experimental section}

EBIE was performed at room temperature using a FEI Nova NanoSEM variable pressure ${ }^{38}$ scanning electron microscope (SEM) equipped with an environmental sub-chamber described elsewhere. ${ }^{12}$ The substrates were $1.7 \mu \mathrm{m}$ films of UNCD grown on silicon by hot filament chemical vapor deposition $(\mathrm{HFCVD})$ at $953 \mathrm{~K}$ (grain size $=2-5 \mathrm{~nm}$, average roughness $=10 \mathrm{~nm}$ ). ${ }^{39}$ Samples were annealed in situ for six hours at $573 \mathrm{~K}$ under flowing $\mathrm{H}_{2} \mathrm{O}$ vapor to desorb residual hydrocarbon adsorbates prior to performing EBIE at $300 \mathrm{~K}$ using $13.6 \mathrm{~Pa}$ of $\mathrm{H}_{2} \mathrm{O}$ as the precursor gas. Cylindrical etch pits were fabricated as a function of time using a $20 \mathrm{keV}, 3.4 \mathrm{nA}$, stationary electron beam under-focused to a diameter of $\sim 1.9 \mu \mathrm{m}$ to produce a top-hat flux profile ${ }^{18}$ (see Figure 1). Additional pits were produced using a beam diameter of $\sim 2.0 \mu \mathrm{m}$, beam energies of 5 and $10 \mathrm{keV}$ and currents of 2.3 and $3.3 \mathrm{nA}$, respectively (Figure 4). All pits were imaged ex situ using the tapping mode of a Digital Instruments Dimension 3100 atomic force microscope (AFM), and analyzed using the software package Gwyddion. ${ }^{40}$ As-grown, H-terminated UNCD was hydrophobic, with a water contact angle $\theta_{c}$ of $\sim 85^{\circ}$. Hydrophilic UNCD $\left(\theta_{c} \sim 8^{\circ}\right.$, measured 
in air after samples were removed from the SEM) was produced by oxygen plasma processing ${ }^{41,42}$ performed in situ ${ }^{43}$ for 2.5 hours, using a XEI Scientific Evactron installed on the SEM used for EBIE $\left(\mathrm{RF}\right.$ power $=13 \mathrm{~W}, \mathrm{O}_{2}$ pressure $\left.=40 \mathrm{~Pa}\right)$. Error bars in Figures 2 and 4(a) account for measurement uncertainty and are dominated by the effects of surface roughness on AFM image analysis.

\section{Results and Discussion}

\section{Surface site activation}

The case of active sites on a passive surface can be incorporated in model \#1 by multiplying Eq. (2) by $A_{s} N_{s}$, where $N_{s}$ and $A_{s}$ are the concentration and area of an active site, respectively:

$$
\frac{\partial N_{\alpha}}{\partial t}=\sigma_{a} f N_{a} A_{s} N_{s}
$$

If $N_{s}$ changes with time, as in the case of cumulative radiation damage occurring during EBIE, then:

$$
\frac{\partial N_{s}}{\partial t}=C\left(1-A_{s} N_{s}\right)
$$

where $C$ is the surface site activation flux $\left(\mathrm{m}^{-2} \mathrm{~s}^{-1}\right)$ and $\left(1-A_{S} N_{S}\right)$ limits the concentration of active sites to one monolayer. $A_{s} N_{s}$ is the fraction of sites that are chemically active and can be volatilized by the fragments $\alpha$, and $\left(1-A_{S} N_{S}\right)$ is the corresponding fraction of $\alpha$ which are generated by the electron beam but do not contribute to etching. These fragments are assumed to leave the substrate through desorption (e.g., $\mathrm{O}(\mathrm{a}) \rightarrow \mathrm{O}(\mathrm{g})$, and $\mathrm{O}(\mathrm{a})+\mathrm{O}(\mathrm{a}) \rightarrow \mathrm{O}_{2}(\mathrm{~g})$ ). Consequently, $\sigma_{a}$

is the true adsorbate dissociation cross-section, ${ }^{23}$ rather than the 'effective' reaction cross-section $\sigma_{\alpha}$ used in Eq. (2), which can be redefined as:

$$
\sigma_{\alpha} \equiv \sigma_{a} A_{s} N_{s}
$$


We note, however, that the above definition of an effective cross-section is less meaningful than Eq. (4) since $N_{s}$ can change with time, whereas EBIE models are normally based on scattering cross-sections which depend only on the species of the adsorbate $a$, and the substrate surface. Hence, we define model \#2 by Eqs. (1) and (3) to (5), and use Eq. (6) merely to illustrate a shortcoming of standard EBIE models.

A consequence of Eq. (5) that is experimentally verifiable and unique to model \#2 is that the etch rate can increase with time during EBIE. Below, we demonstrate such an increase by $\mathrm{H}_{2} \mathrm{O}$-mediated EBIE of the electron sensitive material UNCD. The observed behavior can not be explained by standard EBIE models. It reveals a novel EBIE regime that is rate limited by a growing concentration of active sites, and is distinct from the electron flux and precursor mass transport limited regimes ${ }^{1-3,18,44}$ documented in the literature.

\section{Electron beam induced etching of UNCD}

Figure 2 shows plots of etch pit depth versus time measured from hydrophobic and hydrophilic UNCD. The initial vertical etch rate $\left(\partial z_{d} / \partial t\right)$ is negligible and increases in both cases over the entire time scale (60 to $1440 \mathrm{~s}$ ) probed by the experiments. Figure 2 (a) also shows a datapoint from an etch process that was performed for $8 \mathrm{~min}$, interrupted for $15 \mathrm{~min}$ and resumed for $7 \mathrm{~min}$. The resulting depth is the same as that of an uninterrupted 15 min etch process, showing that the change in etch rate is not reversible over the experimental time scale. The etch rate per unit electron flux was the same in all cases and did not change with small changes in beam diameter. From these results we can conclude that residual hydrocarbons, ${ }^{12,45,46}$ hydrophobicity, variations in adsorbate concentration and beam heating are not primary causes of the observed super-linear $z_{d}(t)$ profiles, and that adsorbate depletion ${ }^{1-3,18}$ was negligible during EBIE (i.e., the rate was not limited by mass transport of precursor molecules into the etched region of the substrate).

Conventional EBIE models (i.e., model \#1) can not reproduce the measured super-linear $z_{d}(t)$ profiles seen in Figure 2. The models predict an initial decrease in $N_{a}$ that typically lasts $\sim$ $10^{-3} \mathrm{~s},{ }^{47}$ followed by constant, steady state etching over the time scale of a typical etch pit fabri- 

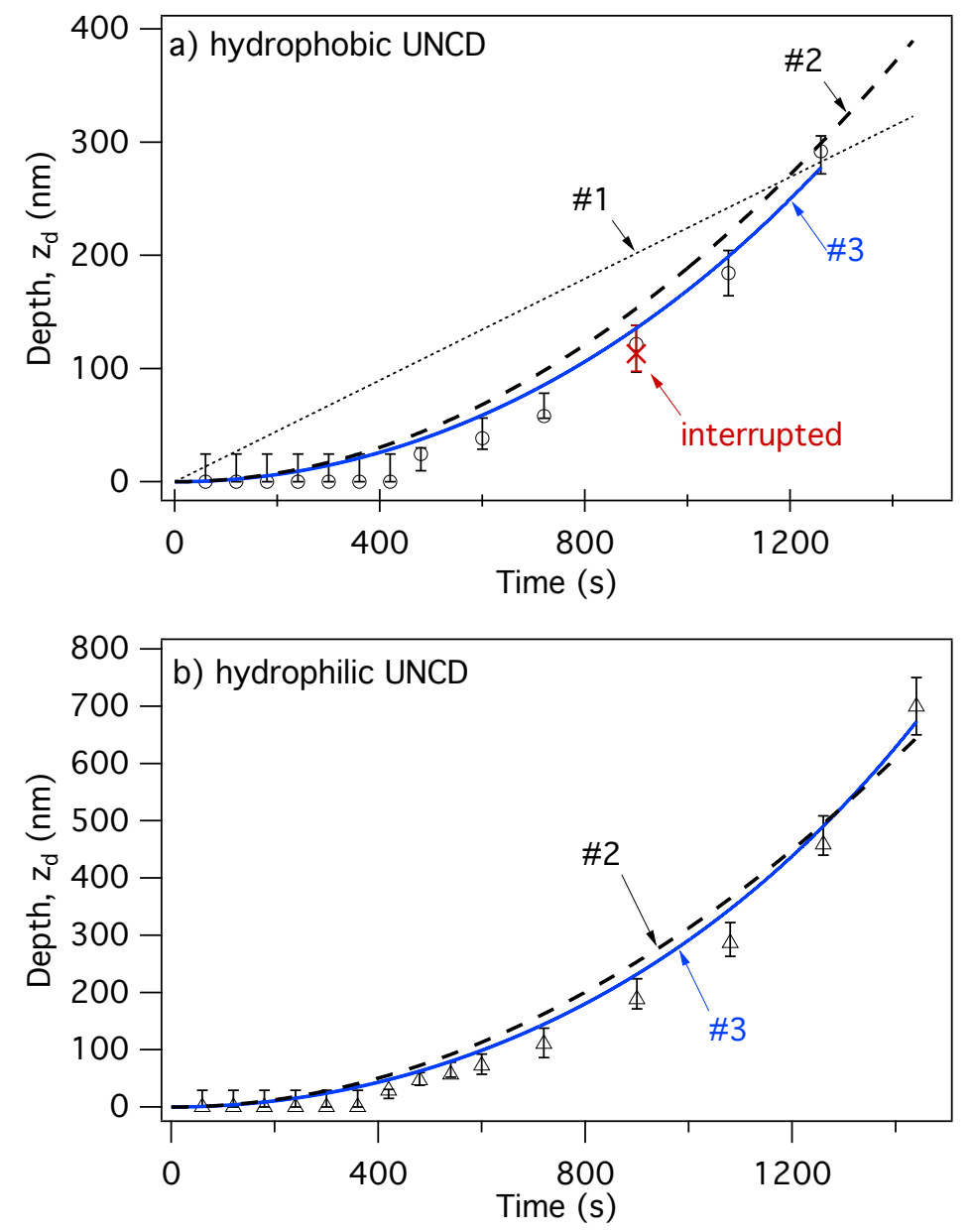

Figure 2: Etch pit depth $\left(z_{d}\right)$ versus time $(t)$ measured from (a) hydrophobic $(\circ$, beam diameter $=$ $2 \mu \mathrm{m})$ and $(\mathrm{b})$ hydrophilic $(\triangle$, beam diameter $=1.8 \mu \mathrm{m})$ UNCD. Also shown is a datapoint $[\times]$ from a process that was interrupted for $15 \mathrm{~min}$, and curves calculated using models \#1, \#2 and \#3 $\left(\sigma_{\alpha}=0.2 \AA^{2}, \sigma_{\mathrm{a}}=2.8 \AA^{2}\right)$. The difference in vertical etch rate between (a) and (b) is caused by the difference in electron beam diameter (the etch rate per unit electron flux is the same in both cases).

cation process $\left(\sim 10^{1}\right.$ to $\left.10^{3} \mathrm{~s}\right) .{ }^{48}$ That is, model \#1 predicts $z_{d}(t)$ profiles such as the one shown in Figure 2(a), which are linear over the experimental time scale, and have a slope given by $\sigma_{\alpha}$.

The measured data are, however, qualitatively consistent with model \#2. This is illustrated in Figure 2 by curves obtained by treating $\sigma_{a}$ and $C$ as fitting parameters which determine the amplitude and curvature of the calculated $z_{d}(t)$ profiles. In both cases (i.e., hydrophobic and hydrophilic UNCD), best fit was obtained by setting $\sigma_{a}$ to $2.8 \AA^{2}$ and $C / f$ to $6 \times 10^{-7}$ (active sites per electron injected into the substrate). 
The etch behavior predicted by model \#2 is in reasonable agreement with experiment. However, the model is based on the simplifying assumption that $C$ does not change with time during etching. This assumption is incorrect for the case of damage produced by an electron beam because the electrons have a maximum penetration range $R_{e}$ in the substrate (shown in Figure 3), and defects are generated at different rates throughout the electron-solid interaction volume. Hence, in the following, we develop 'model \#3' which accounts for both the depth and the time dependence of the defect generation rate in the UNCD substrate, and for the fact that the etched surface recedes during EBIE.

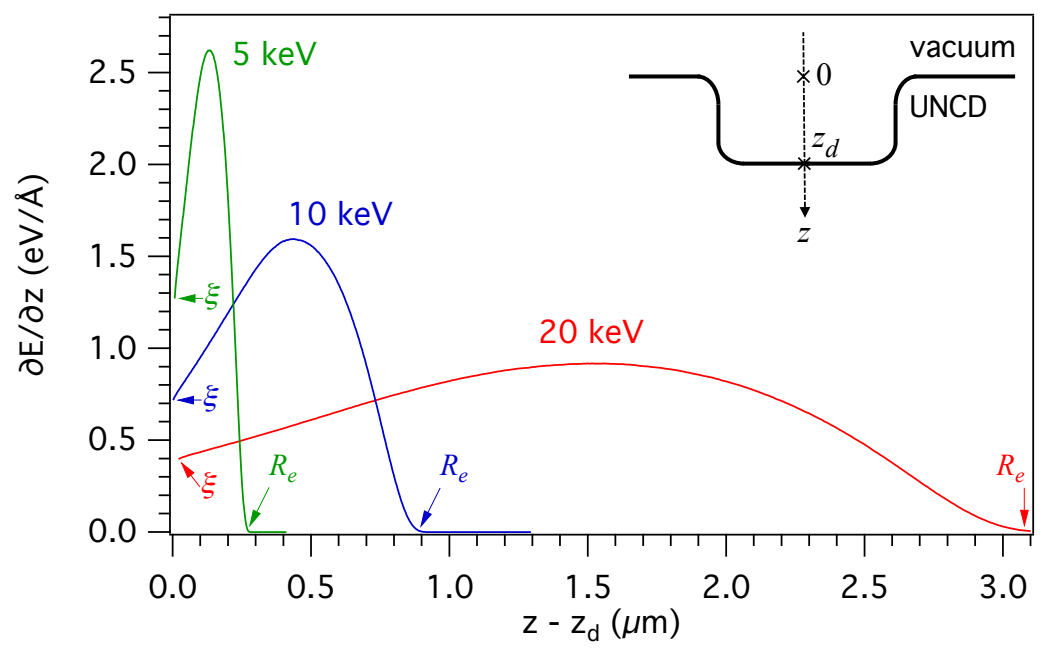

Figure 3: Electron energy deposition profile, $\partial E / \partial z$, calculated for UNCD using electron energies $E_{0}$ of 5,10 and $20 \mathrm{keV}\left(R_{e}=\right.$ maximum electron energy penetration range, $\xi=\partial E / \partial z$ in the limit $[t \rightarrow 0])$ Inset: Schematic illustration of an etch pit and the co-ordinate system used in model \#3.

\section{Generation of chemically active defects during EBIE}

Electron induced defect generation is initiated through two general mechanisms: (i) knock-on caused by momentum transfer from electrons to nuclei, and (ii) bond breaking, ionization and excitation caused by electron-electron scattering. ${ }^{24-26,26-31,49}$ The latter dominate at low electron energies (such as that used here), and the electron-electron scattering rate is approximately proportional to the total electron energy density, ${ }^{24,25,50,51} \frac{\partial E}{\partial V}\left(\mathrm{eV} / \mathrm{m}^{3}\right)$, deposited by the beam into each point $(x, y, z)$ in the solid. However, in the present case of a broad, top-hat, stationary electron 
beam (Figure 1(d)), $\frac{\partial E}{\partial V}$ is approximately constant at each value of $z$ (i.e., across the etch pit, in the plane of the substrate surface). We therefore ignore proximity effects at the etch pit periphery, and approximate the energy deposition profile with $\frac{\partial E}{\partial z}$, the energy deposited into the substrate per unit distance per electron $(\mathrm{eV} / \mathrm{m})$. The deposited energy varies with depth as shown in Figure 3 for electron beam energies of 5, 10 and $20 \mathrm{keV}$, and is assumed to be independent of $x$ and $y$ within the diameter of each etch pit (it was calculated for UNCD using standard Monte Carlo models ${ }^{51,52}$ of electron-solid interactions).

Hence, local defect generation at each point $(x, y, z)$ within the electron-solid interaction volume can be described by:

$$
\frac{\partial K}{\partial t}=n \frac{\partial E}{\partial z} f\left(1-V_{s} K\right)
$$

where $K$ the local defect concentration $\left(\mathrm{m}^{-3}\right)$ which is a function of $z$ and $t, \frac{\partial K}{\partial t}$ is defect generation flux (defects $/ \mathrm{m}^{3} / \mathrm{s}$ ), and $n$ is the number of defects generated per unit energy deposited into the substrate $\left(\mathrm{eV}^{-1}\right)$. That is, $n \frac{\partial E}{\partial z}$ is the number of defects generated in the solid per unit energy deposited into the substrate, and $V_{s}$ is the volume of a single defect (and corresponds to $A_{S}$ ).

We can now complete model \#3 by incorporating the expression $K(z, t)$ into model \#2 by redefining $N_{s}$ as:

$$
N_{s}=\int_{z_{d}}^{z_{d}+z_{\gamma}} K d z
$$

where $z_{\gamma}$ is the thickness of one monolayer of the substrate (and corresponds to $V_{\gamma}$ in Eqs. (3) and (7)). Figure 2 shows the best fit to experiment obtained using model \#3, defined by Eqs. (1), (3), (4), (7) and (8). The model input parameters were those used in model \#2, the $20 \mathrm{keV} \frac{\partial E}{\partial z}$ profile shown in Figure 3, and the coordinate system shown in the inset of Figure 3. The fitting parameter $n$ was set to $1.35 \mathrm{MeV}^{-1}$ (i.e., 135 defects per $100 \mathrm{MeV}$ deposited into the substrate). The resulting $z_{d}(t)$ profile is in better agreement with experiment than model \#2 because $\frac{\partial E}{\partial z}$ increases with $z$ throughout the maximum etch pit depth probed by the experiments (i.e., 292 and $700 \mathrm{~nm}$ in the case of hydrophobic and hydrophilic UNCD, as seen in Figure 2 (a) and (b), respectively). ${ }^{53}$

We note that the value of $\sigma_{\alpha}$ used in model \#1 is much lower than that of $\sigma_{a}$ used in models \#2 
and \#3 ${ }^{23}\left(\sigma_{\alpha}=0.2 \AA^{2}\right.$ and $\left.\sigma_{a}=2.8 \AA^{2}\right)$. This difference is expected since $\sigma_{\alpha}$ accounts only for the dissociation of fragments that lead to etching, as defined by Eq. (6).

To verify the validity of model \#3, we performed an additional experiment in which we analyzed the etch rate at a number of electron beam energies, $E_{0}$. Figure 3 shows $\frac{\partial E}{\partial z}$ profiles calculated for $E_{0}=20,10$ and $5 \mathrm{keV}$. The curves illustrate that, at the surface $[z \rightarrow 0], \frac{\partial E}{\partial z}$ increases as $E_{0}$ is reduced from 20 to $5 \mathrm{keV}$. Hence, based on model \#3, the initial EBIE rate, $\frac{\partial z_{d}}{\partial t}$, should scale accordingly since it is directly proportional to $\frac{\partial E}{\partial z}[z \rightarrow 0]$. To test this prediction, we measured the etch onset time, $t_{e}$, which we defined as the minimum EBIE time needed to detect a pit in AFM images of the substrate. This comparison is appropriate because the initial experimental etch rate was undetectable (over the intrinsic surface roughness of the as-grown UNCD), implying that $t_{e}$ is governed by $N_{s}$ and essentially independent of $\sigma_{a}$. Conversely, a quantitative comparison of the etch rates, $\frac{\partial z_{d}}{\partial t}$, at 5,10 and $20 \mathrm{keV}$ is confounded by the fact that the amplitude of the secondary electron spectrum and hence the value of $\sigma_{a}$ change with $E_{0}{ }^{23}$

Figure 4(a) shows the experimental data obtained using electron beam energies of 5 and $10 \mathrm{keV}$. The results are expressed as the maximum depth detected in the AFM image of each etch pit so as to show how the values compare to the intrinsic surface roughness of the substrate (a sample AFM image and line profile are shown in Figure 4(b)). The figure also shows the etch onset times predicted for 5 and $10 \mathrm{keV}$ using:

$$
t_{e}\left(E_{0}\right)=t_{20} \xi_{20} / \xi_{E_{0}}
$$

where $t_{20}$ is the experimental etch onset time at $20 \mathrm{keV}$ (obtained from the data shown in Figure 2), $\xi_{20}$ is $\frac{\partial E}{\partial z}[t \rightarrow 0]$ at $E_{0}=20 \mathrm{keV}$, and $\xi_{E_{0}}$ are the corresponding values at 5 and $10 \mathrm{keV}$ (shown in Figure 3). The predicted etch onset times are in good agreement with experiment, indicating that the initial rate does indeed scale with the energy density deposited into the near-surface region of the substrate, as predicted by model \#3. 

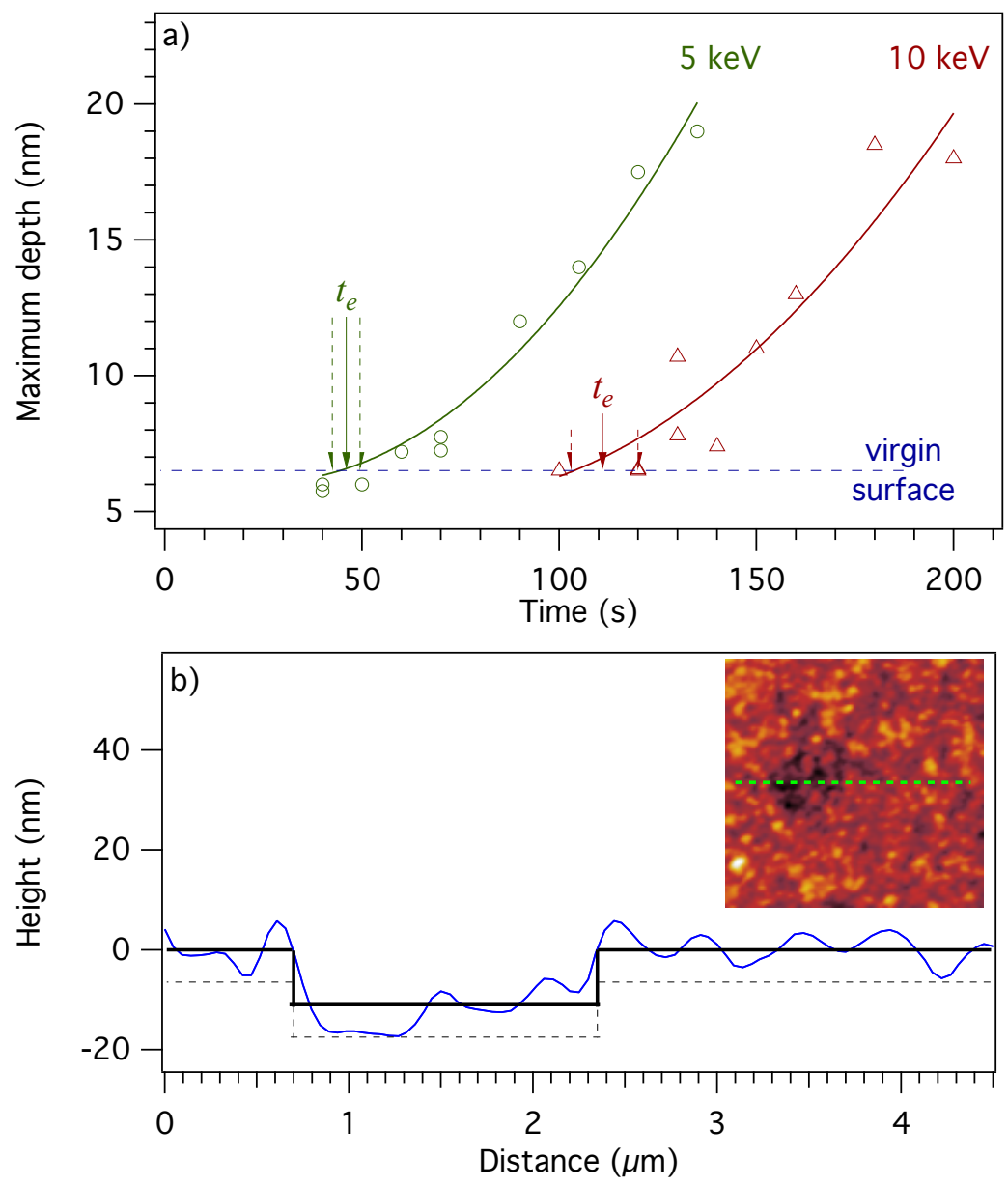

Figure 4: (a) Maximum depth of pits in UNCD fabricated using 5 and $10 \mathrm{keV}$ electron beams, plotted as a function of etch time. The dashed horizontal line indicates the maximum depth measured in adjacent, non-irradiated regions of UNCD, and serves as a measure of initial surface roughness. Arrows labelled $t_{e}$ show the etch onset times predicted using model \#3. Dashed arrows show the corresponding error bars. (b) AFM line profile across a pit fabricated using a beam energy of $5 \mathrm{keV}$ and an etch time of $120 \mathrm{~s}$. The solid and dashes lines show the location of the etch pit and the substrate surface determined using the minimum and average depths measured inside and near the etch pit. Inset: AFM image and the position of the line scan used to generate the plot in (b).

\section{Conclusions}

We have incorporated dynamic surface site activation and the role of electron beam damage into models of EBIE rate kinetics. The refined models yield higher order rate kinetics, predict a new rate kinetics regime limited by the concentration of active surface sites, $N_{s}$, and reduce to standard EBIE models when the active site coverage approaches unity (i.e., $A_{s} N_{s} \rightarrow 1$ ). The refined models are in good agreement with experiments which indicate that EBIE of UNCD proceeds through an 
electron restructuring pathway. Analogous restructuring effects likely play a role in EBIE of other materials, and possibly account for atypical dependencies of etch rate on time which have been reported previously for a number of precursor-substrate combinations. ${ }^{5,11,54}$ The results presented here have implications for the construction of predictive EBIE models, ultimate resolution and proximity effects inherent to EBIE.

\section{Acknowledgement}

This work was funded by FEI Company and the Australian Research Council. Aiden Martin is the recipient of a John Stocker Postgraduate Scholarship from the Science and Industry Endowment Fund.

\section{References}

(1) Randolph, S. J.; Fowlkes, J. D.; Rack, P. D. Crit. Rev. Solid State Mater. Sci. 2006, $31,55$.

(2) Utke, I.; Hoffmann, P.; Melngailis, J. J. Vac. Sci. Technol., B: Nanotechnol. Microelectron.: Mater., Process., Meas., Phenom. 2008, 26, 1197.

(3) Utke, I.; Moshkalev, S.; Russell, P. Nanofabrication Using Focused Ion and Electron Beams: Principles and Applications; Oxford University Press: New York, 2012.

(4) Arumainayagam, C. R.; Lee, H.-L.; Nelson, R. B.; Haines, D. R.; Gunawardane, R. P. Surf. Sci. Rep. 2010, 65, 1.

(5) Toth, M.; Lobo, C.; Knowles, W.; Phillips, M.; Postek, M.; Vladár, A. Nano Lett. 2007, 7 , 525.

(6) Perry, J. M.; Harms, Z. D.; Jacobson, S. C. Small 2012, 8, 1521.

(7) Thiele, C.; Engel, M.; Hennrich, F.; Kappes, M. M.; Johnsen, K.-P.; Frase, C. G.; Loehneysen, H. V.; Krupke, R. Appl. Phys. Lett. 2011, 99, 173105. 
(8) Goler, S.; Piazza, V.; Roddaro, S.; Pellegrini, V.; Beltram, F.; Pingue, P. J. Appl. Phys. 2011, $110,064308$.

(9) Spinney, P. S.; Howitt, D. G.; Collins, S. D.; Smith, R. L. Nanotechnology 2009, 20, 465301.

(10) Wang, D.; Hoyle, P.; Cleaver, J.; Porkolab, G.; Macdonald, N. J. Vac. Sci. Technol., B: Nanotechnol. Microelectron.: Mater., Process., Meas., Phenom. 1995, 13, 1984.

(11) Miyazoe, H.; Utke, I.; Michler, J.; Terashima, K. Appl. Phys. Lett. 2008, 92, 043124.

(12) Lobo, C. J.; Martin, A.; Phillips, M. R.; Toth, M. Nanotechnology 2012, 23, 375302.

(13) Hoffmann, P.; Utke, I.; Perentes, A.; Bret, T.; Santschi, C.; Apostolopoulos, V. Proc. SPIE 2005, 592506.

(14) Niitsuma, J.; Yuan, X.; Koizumi, S.; Sekiguchi, T. Jpn. J. Appl. Phys., Part 2 2006, 45, L71.

(15) Taniguchi, J.; Miyamoto, I.; Ohno, N.; Kantani, K.; Komuro, M.; Hiroshima, H. Jpn. J. Appl. Phys. 1997, 36, 7691.

(16) Yao, N. Focused Ion Beam Systems: Basics and Applications; Cambridge University Press: Cambridge, 2011.

(17) Lobo, C. J.; Toth, M.; Wagner, R.; Thiel, B. L.; Lysaght, M. Nanotechnology 2008, 19, 025303.

(18) Randolph, S.; Toth, M.; Cullen, J.; Chandler, C.; Lobo, C. Appl. Phys. Lett. 2011, 99, 213103.

(19) Lassiter, M. G.; Rack, P. D. Nanotechnology 2008, 19, 455306.

(20) Fowlkes, J. D.; Rack, P. D. ACS Nano 2010, 4, 1619.

(21) Smith, D. A.; Fowlkes, J. D.; Rack, P. D. Small 2008, 4, 1382.

(22) Bishop, J.; Lobo, C. J.; Martin, A.; Ford, M.; Phillips, M. R.; Toth, M. Phys. Rev. Lett. 2012 , $109,146103$. 
(23) The values of $\sigma_{\alpha}$ and $\sigma_{a}$ used in the models represent cross-sections weighed by the overlap between the corresponding energy dependent cross-section and energy spectra of electrons incident onto and emitted from the substrate ${ }^{3}$

(24) Krasheninnikov, A. V.; Nordlund, K. J. Appl. Phys. 2010, 107, 071301.

(25) Egerton, R. F.; Li, P.; Malac, M. Micron 2004, 35, 399.

(26) Krasheninnikov, A. V.; Banhart, F. Nat. Mater. 2007, 6, 723.

(27) Banhart, F. Rep. Prog. Phys. 1999, 62, 1181.

(28) Kuznetsov, V.; Chuvilin, A.; Moroz, E.; Kolomiichuk, V.; Shaikhutdinov, S.; Butenko, Y.; Malkov, I. Carbon 1994, 32, 873.

(29) Teweldebrhan, D.; Balandin, A. A. Appl. Phys. Lett. 2009, 94, 013101.

(30) Liu, G.; Teweldebrhan, D.; Balandin, A. A. IEEE Trans. Nanotechnol. 2011, 10, 865.

(31) Barjon, J.; Mehdaoui, A.; Jomard, F.; Chevallier, J.; Mer, C.; Nesladek, M.; Bergonzo, P.; Pernot, J.; Omnes, F.; Deneuville, A. Appl. Phys. Lett. 2008, 93, 062108.

(32) Bello, I.; Fung, M.; Zhang, W.; Lai, K.; Wang, Y.; Zhou, Z.; Yu, R.; Lee, C.; Lee, S. Thin Solid Films 2000, 368 (2), 222.

(33) Yang, R.; Zhang, L.; Wang, Y.; Shi, Z.; Shi, D.; Gao, H.; Wang, E.; Zhang, G. Adv. Mater. 2010, 22, 4014 .

(34) Carlsson, J. M.; Hanke, F.; Linic, S.; Scheffler, M. Phys. Rev. Lett. 2009, 102, 166104.

(35) Bachmann, P.; Leers, D.; Wiechert, D. Diamond Relat. Mater. 1993, 2, 683.

(36) Dickrell, P. L.; Argibay, N.; Eryilmaz, O. L.; Erdemir, A.; Sawyer, W. G. J. Tribol. 2009, 131, 032102.

(37) Hagymassy, J., Jr.; Brunauer, S.; Mikhail, R. S. J. Colloid Interface Sci. 1969, 29, 485-491. 
(38) Danilatos, G. D. Adv. Electron. Electron Phys. 1988, 71, 109.

(39) Adiga, V.; Sumant, A.; Suresh, S.; Gudeman, C.; Auciello, O.; Carlisle, J.; Carpick, R. Phys. Rev. B: Condens. Matter Mater. Phys. 2009, 79, 245403.

(40) Nečas, D.; Klapetek, P. Cent. Eur. J. Phys. 2012, 10, 181.

(41) Ostrovskaya, L.; Perevertailo, V.; Ralchenko, V.; Dementjev, A.; Loginova, O. Diamond Relat. Mater. 2002, 11, 845.

(42) Popov, C.; Kulisch, W.; Bliznakov, S.; Ceccone, G.; Gilliland, D.; Sirghi, L.; Rossi, F. Diamond Relat. Mater. 2008, 17, 1229.

(43) Vladar, A.; Postek, M.; Vane, R. Proc. SPIE 2001, 4344, 835.

(44) White, W. B.; Rykaczewski, K.; Fedorov, A. G. Phys. Rev. Lett. 2006, 97, 086101.

(45) Toth, M.; Lobo, C. J.; Hartigan, G.; Ralph Knowles, W. J. Appl. Phys. 2007, 101, 054309.

(46) Toth, M.; Lobo, C. J.; Lysaght, M. J.; Vladar, A. E.; Postek, M. T. J. Appl. Phys. 2009, 106, 034306.

(47) The initial decrease corresponds to the transition from initial $\left(N_{a}[t=0]\right)$ to steady state $\left(N_{a}[t \rightarrow \infty]\right)$ at the start of an etch process. It is caused by adsorbate consumption in the etch reaction, ${ }^{3}$ and was negligible under the conditions used here (i.e., $N_{a}[t \rightarrow \infty] / N_{a}[t=0] \approx 1$ ).

(48) An additional decrease in etch rate with time has been reported in the case of high aspect ratio pits and mass transport limited EBIE. ${ }^{18}$ It is caused by a decrease in the conductance of a growing etch pit during EBIE, and can not explain the super-linear $z_{d}(t)$ profiles reported here.

(49) Meyer, J. C.; Eder, F.; Kurasch, S.; Skakalova, V.; Kotakoski, J.; Park, H. J.; Roth, S.; Chuvilin, A.; Eyhusen, S.; Benner, G.; Krasheninnikov, A. V.; Kaiser, U. Phys. Rev. Lett. 2012, 108, 196102. 
(50) Everhart, T.; Hoff, P. J. Appl. Phys. 1971, 42, 5837.

(51) Toth, M.; Phillips, M. R. Scanning 1998, 20, 425.

(52) Hovington, P.; Drouin, D.; Gauvin, R. Scanning 1997, 19, 1.

(53) Based on model \#3, a steady state (whereby $\partial N_{s} / \partial t=0$ at the etch pit/vacuum interface) will be reached if the sample thickness and the etch pit depth are greater than the electron penetration range.

(54) Randolph, S. J.; Fowlkes, J. D.; Rack, P. D. J. Appl. Phys. 2005, 98, 034902. 
Graphical TOC Entry

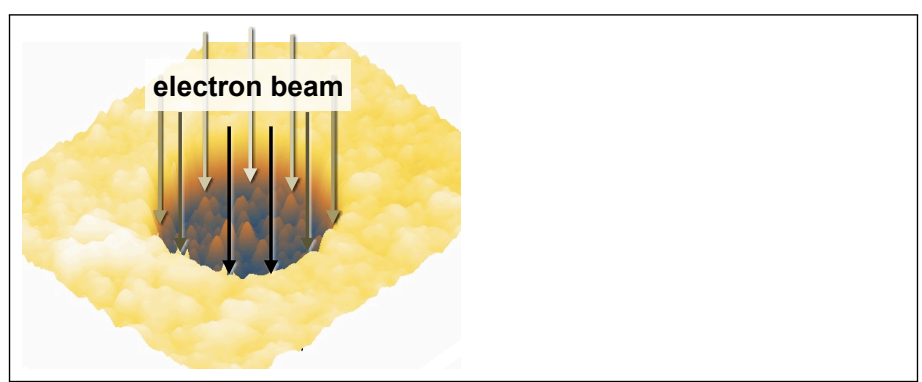

